\title{
Concurrent CCR7 Overexpression and RelB Knockdown in Immature Dendritic Cells Induces Immune Tolerance and Improves Skin-Graft Survival in a Murine Model
}

\author{
Zhiwei Dong ${ }^{a}$ Yajie Chen ${ }^{a}$ Yuan Peng ${ }^{c}$ Fan Wang ${ }^{d}$ Zichen Yang ${ }^{a}$ \\ Guangtao Huang Yu Chen ${ }^{\mathrm{a}}$ Zhiqiang Yuan $^{\mathrm{a}}$ Tongtong Cao $^{\mathrm{b}}$ Yizhi Peng $^{\mathrm{a}}$ \\ Institute of Burn Research, Southwest Hospital, State Key Laboratory of Trauma, Burns and Combined \\ Injury, Third Military Medical University, Chongqing, ' ${ }^{\circ}$ Department of Pathology, Shanghai University \\ of Traditional Chinese Medicine, Shanghai, 'The first clinical college of Chongqing Medical University, \\ Chongqing, ${ }^{\mathrm{d}}$ Department of Plastic and Reconstructive Surgery of Southwest Hospital, Third Military \\ Medical University Chongqing, China
}

\section{Key Words}

imDCs • Treg cells $\bullet$ CCR7 $\bullet$ RelB $\bullet$ Immune tolerance $\bullet$ Transplantation

\begin{abstract}
Background/Aims: Skin transplantation aims to cover skin defects but often fails due to immune rejection of the transplantated tissue. Immature dendritic cells (imDCs) induce immune tolerance but have a low migration rate. After stimulation, imDCs transform into mature DCs, which activate immune rejection. Thus, inducing imDC to obtain a high migration counteracts development of immune tolerance. Methods \& Results: We transfected imDCs with a recombinant adenovirus carrying the CCR7 gene (Ad-CCR7) and a small interfering RNA targeting RelB (RelB-siRNA) to concurrently overexpress CCR7 and downregulate RelB expression. Functionally, such cells showed a significantly enhanced migration rate in the chemotactic assay and decreased T-cell proliferation after lipopolysaccharide stimulation in mixed lymphocyte reactions. Cotransfected cells showed an increased ability to induce immune tolerance by upregulating $T$ regulatory (Treg) cells and shifting the Th1/Th2 ratio. Cotransfection of Ad-CCR7 and RelB-siRNA endowed imDCs with resistance to apoptosis and cell death. CCR7 overexpression and RelB knockdown (KD) in imDCs improve skin-graft survival in a murine skin-transplantation model. Conclusion: Transfection with Ad-CCR7 and ReIB KD in imDCs may be an effective approach inducing immune tolerance, thus being potentially valuable for inhibiting allograft rejection.

\section{Introduction}

Allogeneic skin transplantation is an early, effective method used to cover wounded areas affected by large deep burns or other forms of trauma, as well as a promising approach 


\section{Cellular Physiology Cell Physiol Biochem 2017;42:455-468 \\ \begin{tabular}{l|l} 
DOI: 10.1159/000477593 & Ond Biochemistry \\
Published online:;Vay 31, 2017 & $\begin{array}{l}\text { 2017 The Author(s). Published by S. Karger AG, Basel } \\
\text { www.karger.com/cpb }\end{array}$
\end{tabular} \\ Dong et al.: Dendritic Cells Induce Immune Tolerance and Improve Skin-Graft Survival}

to circumvent the current skin shortage [1]. However, organ rejection is the greatest barrier to successful xenotransplantation, which substantially jeopardizes skin-graft survival. It has previously been reported that xenograft survival is longer in CD4+ T-cell-depleted mice than in CD4+ T-cell mice. Overexpression of inhibitory molecules, including interleukin (IL)-10, transforming growth factor (TGF)-b, and suppressor of cytokine signaling (SOCS) 1, can also induce tolerance in dendritic cells (DCs) (Tol-DC), thus inhibiting immune rejection more efficiently. Some cytokines and drugs can be used as immune-tolerance inducers by affecting the immune ability of the antigen-presenting cells (APC) and naive T cells [2], suggesting that cell-mediated immune responses play a critical role in successful xenotransplantation of skin.

It is important that factors in immune tolerance, including the characteristics of immune cells, particularly APCs and regulatory T cells (Tregs), are explored to enhance skin-graft survival and function. DCs are specialized APCs which play an important role in immune tolerance and immunological rejection. Immature DCs (imDCs) can generate immune tolerance because of their relatively lower antigen-presentation ability and the need for fewer costimulatory molecules. However, imDCs have a relatively low expression of C-C chemokine receptor type 7 (CCR7), which regulates DC migration, resulting in the slow imDC migration, possibly affecting immune-tolerance ability. In our previous research, we attempted to increase CCR7 expression by adenovirus transfection in imDCs to obtain a high migration ability [3]. A major limitation of adenovirus transfection is that it will also induce imDCs to partially differentiate into mDCs, thereby increasing the possibility of immune rejection. In addition, imDCs are likely to encounter inflammatory stimuli at some stage that will trigger their terminal maturation of DCs $[4,5]$. Therefore, a mechanism to induce the imDC to migrate faster while maintaining immune-tolerance ability is critical for the induction of long-term immune tolerance in vivo.

RelB is a nuclear factor (NF)- $\kappa B$ family member that can regulate the terminal stages of DC maturation by controlling the expression of the necessary costimulatory molecules. A large percentage of previous studies has revealed that RelB is directly associated with the biological function of DCs, and it has been identified as an important transcription factor in immune-regulatory pathways [6, 7]. RelB gene knockdown (RelB KD) decreases costimulatory molecule expression by DCs. DCs isolated from RelB-/- mice show a lack of typical costimulatory molecules and induce antigen-specific regulatory $\mathrm{T}$ cells in vivo, thereby preventing specific immune rejections and enhancing graft longevity [8]. Therefore, the RelB gene is an ideal target for manipulating the tolerance of DCs to control immune rejections. However, the effects of concurrent CCR7 overexpression and RelB KD on the biological functions of imDC, such as migration, immune tolerance, antiapoptosis, and production of key cytokines, have not yet been reported. In addition, it is unclear whether the cotransfection of Ad-CCR7 and RelB small interfering RNA (siRNA) in imDCs can prolong allogenic skin-graft survival.

In the present study, we transfected imDCs with both Ad-CCR7 and RelB-siRNA to investigate changes in biological function of imDCs, and whether this can influence skingraft survival.

\section{Materials and Methods}

Culture of bone-marrow DCs (BMDCs)

Bone-marrow cells were collected from tibiae and femora of C57BL/6 mice. DCs were adjusted to 105 cells/well and cultured in full Roswell Park Memorial Institute (RPMI)-1640 supplemented with 10\% fetal bovine serum (FBS), 20-ng/mL recombinant mouse granulocyte macrophage colony-stimulating factor (rmGM-CSF; Peprotech, Inc., NJ, USA), and 10-ng/mL rmIL-4 (Peprotech) in a 24-well plate. RelBsiRNA was transfected on day 3 and Ad-CCR7 was transfected on day 4 . After 6 days of culture, $1-\mu \mathrm{g} / \mathrm{mL}$ lipopolysaccharide (LPS; Sigma-Aldrich, shanghai, china) was added, and the nonadherent imDCs were harvested on day 7 for use in the experiment. 


\section{Cellular Physiology Cell Physiol Biochem 2017;42:455-468 \\ \begin{tabular}{l|l} 
DOI: 10.1159/000477593 & Ond 2017 The Author(s). Published by S. Karger AG, Basel \\
www.karger.com/cpb
\end{tabular} \\ Dong et al.: Dendritic Cells Induce Immune Tolerance and Improve Skin-Graft Survival}

Transfection of adenovirus and siRNA

The siRNA sequences used for targeting RelB or control sequences were as follows: siRNA-RelB, TGCCGAATCAACAAGGAGA; non-silencing siRNA, TTCTCCGAACGTGTCACGT; all siRNAs were synthesized and annealed by Shanghai GeneChem Limited Company (Shanghai, China). According to the manufacturer's protocol [9], 200-pmol siRNA labeled with 5'-Cy3 was transfected when DCs were 50\%-70\% confluent on day 3 using GeneSilencer® Transfection Reagent (CA, USA). On day 1 after the siRNA transfection (day 4), the cells were transfected with Ad-CCR7 containing green fluorescent protein (GFP); the recombinant vector was constructed in our laboratory using standard protocols [3]. For some experiments, DCs were cultured with LPS $(1 \mu \mathrm{g} / \mathrm{mL})$ for $48 \mathrm{~h}$ on day 6 to allow for maturation. Empty adenoviral vector and scrambled siRNA were transfected as a control. Transduction efficiency was measured by the frequency of GFP-positive or red fluorescent protein (RFP)-positive cells. The nonadherent cells were harvested for detection of RelB, CCR7 protein expression by Western blotting or phenotype detection by fluorescence-activated cell sorting (FACS) at different times.

\section{Flow cytometry}

Phenotype analyses of DCs in the different conditions were evaluated by flow cytometry using the following monoclonal antibodies (mAbs): anti-mouse CD80, CD86, CCR7 (FITC; eBioscience, CA, USA). Briefly, the cells were collected, washed, and incubated at $4^{\circ} \mathrm{C}$ for $30 \mathrm{~min}$ with each mAb diluted to the optimal concentration. The appropriate isotype was used as the control $\mathrm{mAb}$. The cells were subjected to flow cytometric analysis (FACS Calibur flow cytometer).

\section{Cell-migration assay}

Cell-migration assays were performed using a 24-well Transwell microplate with a $5-\mu \mathrm{m}$ membrane pore size (Corning, New York, USA). At day 5, the different types of DCs were resuspended in migration medium (RPMI-1640 supplemented with $1 \%$ human serum, 500-U/mL GM-CSF, and 250-U/mL IL-4), and $200 \mu \mathrm{L}$ of the cell suspension $(2 \times 105$ cells $)$ was added to the upper chamber. The lower chambers contained $600 \mu \mathrm{L}$ migration medium supplemented with 300-ng/mL cCCL19 (R\&D Systems, MN, USA). The chamber was then cultivated in $5 \% \mathrm{CO} 2$ at $37^{\circ} \mathrm{C}$ for $4 \mathrm{~h}$, following which the cells in the upper chamber were removed and migrating DCs harvested from the lower chamber were counted.

\section{Detection of apoptosis and viability of DCs}

Two days after LPS $(1 \mu \mathrm{g} / \mathrm{mL}$ ) stimulation (day 8), the cells were cultured in the starvation media (RPMI-1640 supplemented with $20-\mathrm{ng} / \mathrm{mL}$ rmGM-CSF, $10-\mathrm{ng} / \mathrm{mL}$ rmIL-4, and no serum) for $24 \mathrm{~h}$ to induce the apoptosis. To quantify cell viability, $100 \mathrm{~mL}$ of DCs were seeded $(2.5 \times 105$ cells $/ \mathrm{mL})$ with starvation media in 96-well plates, and the MTT (Sangon Biotech shanghai, China) assay was performed according to manufacturer's instructions. Data are presented as cell activity relative to control samples. Alternatively, the cells were assessed by flow cytometry stained with fluorescein-isothiocytanate (FITC)-conjugated annexin $\mathrm{V}$ and PI, according to the manufacturer's instructions.

One-way mixed lymphocyte reaction (MLR)

One day after LPS stimulation (day 7), different types of DCs were resuspended at $1 \times 105$ cells $/ \mathrm{mL}$ in RPMI-1640 for a 30-min incubation with mitomycin C (25 ng/L) and washed twice with RPMI-1640 to serve as stimulators. The nylon wool-purified splenic T cells $(1 \times 106$ cells $/ \mathrm{mL})$ from BALB/c served as responders; DCs were mixed with T cells at a 1:10 ratio. The mixed cultures were established in triplicate in 96-well round-bottomed microculture plates $(200 \mu \mathrm{L} /$ well $)$ and maintained in a complete medium for $72 \mathrm{~h}$ at $37^{\circ} \mathrm{C}$ in $5 \% \mathrm{CO}$. [3H]-TdR ( $1 \mathrm{mCi} /$ well $)$ was added for the final $18 \mathrm{~h}$ of culture. T cells cultured in medium were used as a control. Cells were harvested onto glass-fiber filter disks, and incorporation of [3H]-TdR into DNA was assessed by a Wallac 1450 liquid scintillation counter (LS 6000 TA, Beckman; Fullerton California). Results are expressed as mean cpm \pm standard deviation (SD).

TTreg-cell test

One day after LPS stimulation (day 7), different types of DCs were cocultured with nylon wool-purified splenic T cells from BALB/c; DCs were mixed with T cells at a 1:10 ratio. To examine the population of CD4 + CD25 + Tregs, cocultured cells were stained with FITC-conjugated anti-mouse CD4 antibody (10 $\mu \mathrm{L})$ 


\section{Cellular Physiology Cell Physiol Biochem 2017;42:455-468 \\ \begin{tabular}{l|l} 
DOI: 10.1159/000477593 & Ond Biochemistry \\
Published onlne:1Vay 31, 2017 & $\begin{array}{l}\text { 2017 The Author(s). Published by S. Karger AG, Basel } \\
\text { www.karger.com/cpb }\end{array}$
\end{tabular} \\ Dong et al.: Dendritic Cells Induce Immune Tolerance and Improve Skin-Graft Survival}

and phycoerythrin (PE)-Cy5-conjugated anti-mouse CD25 antibody (10 $\mu \mathrm{L}$; eBioscience, San Diego, USA) according to the manufacturer's infections. In some experiments, cells were permeabilized and fixed with CytoPerm/CytoFix (BD Biosciences), further stained with anti-mouse Foxp3-PE mAb (eBioscience) and FITCconjugated anti-mouse CD4 antibody. Differentiation of Th1 and Th2 cells was confirmed by intracellular cytokine staining of IFN- $\gamma$ and IL-4 upon 5 hours stimulation with $10 \mathrm{nM}$ phorbol- 12 myristate- 13 acetate (PMA) plus $1 \mu \mathrm{M}$ ionomycin in the presence of BD GolgiStop ${ }^{\mathrm{TM}}$ Protein Transport Inhibitor (BD Biosciences) as the protocol. The cells were incubated at $37^{\circ} \mathrm{C}, 5 \% \mathrm{CO} 2$ for $4 \mathrm{~h}$ and then stained with CD4(eBioscience) for $30 \mathrm{~min}$ at $4{ }^{\circ} \mathrm{C}$ in the dark. The cells were washed again and suspended in $0.1 \%$ saponin buffer for 20 min and incubated with anti-IFN- $\gamma$ (BD Biosciences) or anti-IL-4 (BD Biosciences) for $30 \mathrm{~min}$.

\section{Western blotting}

Western blotting was performed as previously described, and the membrane was immunoblotted with the following primary antibodies: mouse RelB (Cell Signaling, USA) and mouse FoxP3 (eBioscience, San Diego, CA). Signal intensity was analyzed by Image Lab ${ }^{\text {TM }}$ Software Version 4.0.1 (Biorad, Hercules, CA).

Enzyme-linked immunosorbent assays (ELISAs)

One day after LPS stimulation (day 7), different types of DCs were cocultured with nylon wool-purified splenic T cells from BALB/c. After 3 days of coculture, supernatants were collected for IL-4, IL-10, IL-2, and tumor necrosis factor (TNF)- $\alpha$ measurement using ELISA kits (Biotech Co, Ltd., China). Serum samples were collected from the peripheral blood of all recipient mice. A volume of $100 \mu \mathrm{L}$ of diluted serum sample $(1: 2,000)$ was used for ELISA. All samples were measured in duplicate.

\section{Skin allograft transplantation}

Eight-week-old male C57BL/6 and BALB/c mice were kept under pathogen-free conditions in the animal room of the Southwest Hospital affiliated to the Third Medical Military University. All experiments were performed according to the Animal Ethical Committee guidelines. BALB/c mice were recipients of skin transplants from donor C57BL/6 mice. Skin allograft transplant surgery was performed as previously described [9]. Recipient BALB/c mice were primed intravenously with 106 donors of the various phenotypic BMDCs from C57BL/6 3 days before grafting, maintaining a daily injection after the transplantation until the end of the experiment. Skin survival was monitored daily by visual assessment. On day 10 after transplantation, recipient mice were humanely sacrificed to examine the grafted skin. For histological analysis, samples were fixed in $4 \%$ paraformaldehyde overnight, embedded in paraffin, sectioned, and stained with hematoxylin and eosin (H\&E) for light microscopy. Sections were dewaxed, dehydrated, and then stained using H\&E.

\section{Statistical analysis}

The data were provided as mean \pm SD Analysis of variance was used to assess the statistical significance of differences between three or more groups. Allograft survival was reported as median survival time and the percentage of skin survival was plotted using Kaplan-Meier curves.

\section{Results}

Downregulation of RelB expression and CCR7 overexpression in imDCs by cotransfection

The imDCs were transfected with the RelB-siRNA on day 3 and Ad-CCR7 was transfected with GFP on day 4. To assess the potency of gene transfection, the transfected DCs were identified on day 6 under microscope by observing green fluorescence (AdCCR7) and red fluorescence (RelB-siRNA). As shown in Fig. 1A, most of the imDCs in the view presented both green and red fluorescence, which indicated that both Ad-CCR7 and RelB-siRNA were transfected into the imDCs. Compared with the control group, transfection with CCR7-adenovirus or RelB-siRNA upregulated CCR7 expression before LPS treatment or downregulate RelB expression after LPS treatment, as revealed by Western blotting (Fig. 1B). These results indicated that cotransfection of Ad-CCR7 and RelB-siRNA enhanced CCR7 expression in imDCs and simultaneously decreased RelB expression in the presence of LPS treatment. Considering that RelB will adjust CD80 and CD86 expressions, we also 
Fig. 1. CCR7 overexpression and downregulation of RelB expression in imDCs by cotransfection (A) imDCs were transfected with RelB-siRNA for 3 days and CCR7Ad for 2 days. The efficiencies of the transfection were observed with GFP fluorescence (labeled Ad-CCR7) and RFP fluorescence (labeled RelB-siRNA). Nuclei were stained with DAPI (blue). Most of the imDCs were able to express GFP and RFP together. (B) Western blot analysis showed that the cotransfection of Ad-CCR7 and RelB-siRNA significantly increased the expression of CCR7 imDCs before LPS treatment and decreased RelB expression in imDCs after LPS treatment, compared with the control group. ( ${ }^{* *} \mathrm{P}<0.01,{ }^{*} \mathrm{P}<0.05$ ) (C) Representative quantification of the flow cytometry analysis showed the increased expression of CCR7 and decreased expression of CD80 and CD86 in imDCs with CCR7 overexpression and RelB KD compared with the control group ( $* \mathrm{P}<0.05$, $\left.{ }^{* * *} \mathrm{P} \leq 0.001\right)$.

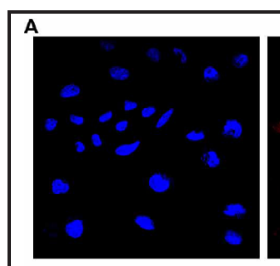

DAPI

B
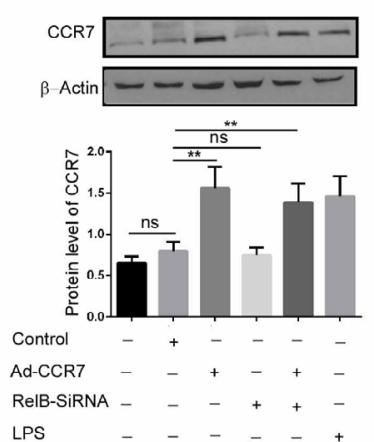

C

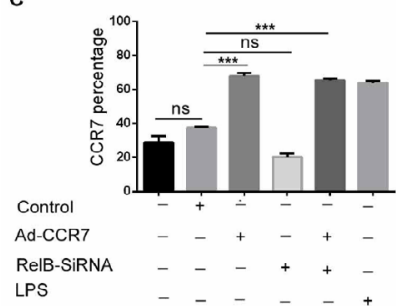

LPS

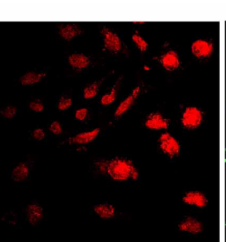

RelB-siRNA

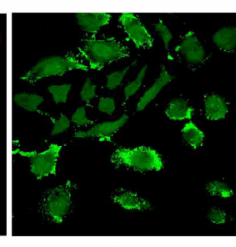

Ad-CCR7
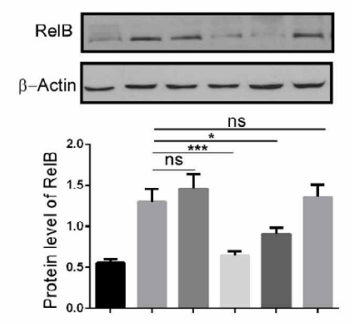

Control

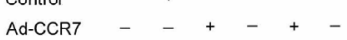

ReIBSIRNA - $-1-0+0+$

LPS -+++++

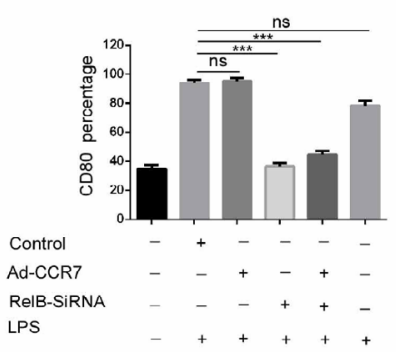

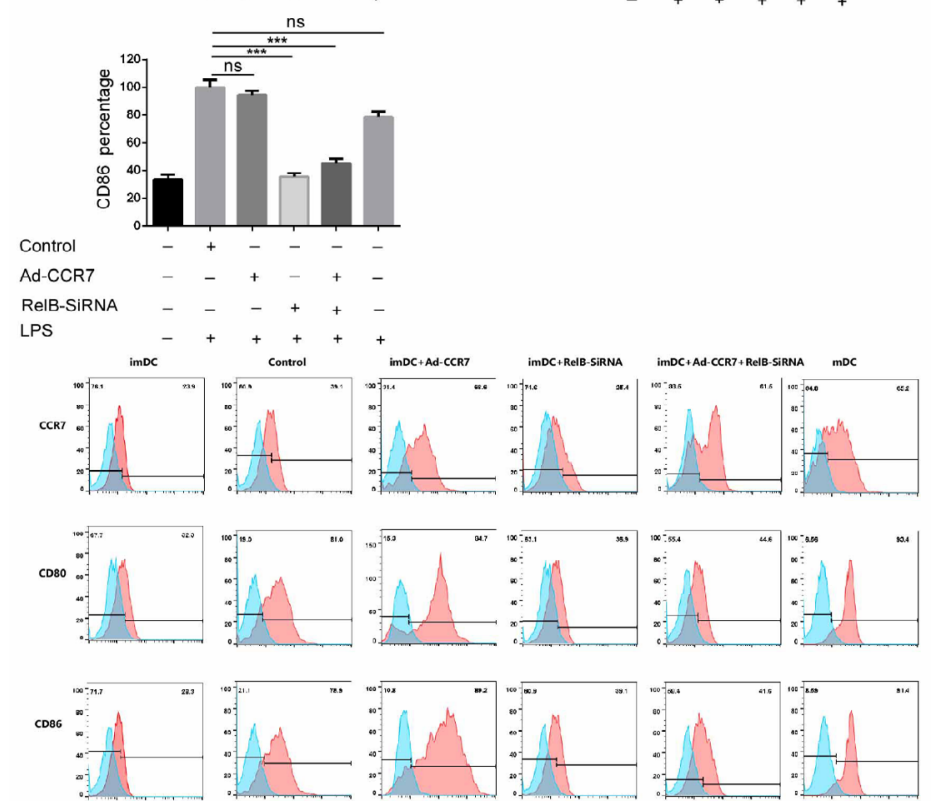

examined CCR7, CD80, and CD86 protein expressions on the surface of DCs by using flow cytometry. As expected, imDCs cotransfected with Ad-CCR7 and RelB-siRNA showed significantly increased CCR7 expression without LPS treatment and significantly decreased CD80 and CD86 expression with LPS stimulation (Fig. 1C). Collectively, the results showed that CCR7 overexpression along with RelB KD in imDCs induced CCR7 overexpression without LPS treatment while maintaining a low expression of costimulatory molecules with LPS treatment. 


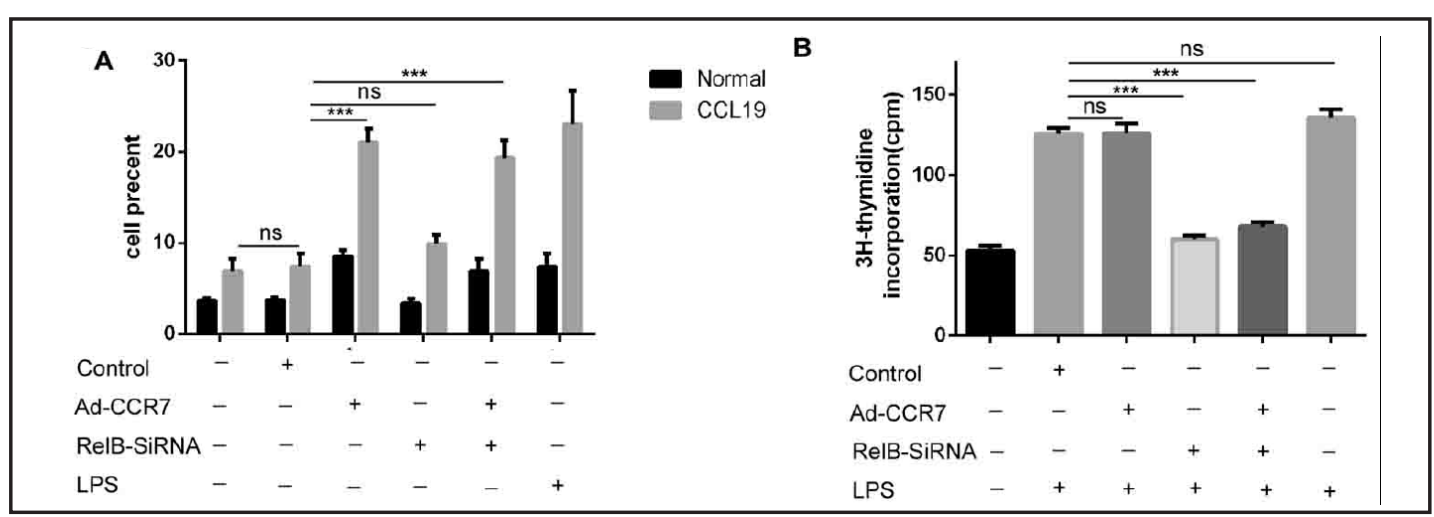

Fig. 2. CCR7 overexpression and RelB KD in imDCs obtained higher migration ability and a lowered ability to stimulate T-cell responses (A) In the presence of CCL19, Transwell migration assay showed that imDCs transfected with both of Ad-CCR7 and RelB-siRNA had higher migration ability. ${ }^{* * *} \mathrm{P}<0.001$, compared with control group. (B) After LPS treatment, the different types of imDCs were cocultured with T cells isolated from BABL/C mice. T-cell proliferation was determined by $3 \mathrm{H}$-thymidine uptake in the mixed lymphocytes reaction (MLR). Results showed that imDCs transfected with both Ad-CCR7 and RelB-siRNA had low abilities to stimulate T-cell proliferation. ${ }^{* * *} \mathrm{P}<0.001$, compared with the control group.

CCR7 overexpression and RelB KD in imDCs obtained high migration ability and lower ability to stimulate T-cell responses

DC migration was mediated by CCR7 in the presence of CCL19. ImDCs have a low migration ability, which could affect their ability to induce immune tolerance [10]. To test the migration ability of the imDCs after transfection, we detected the migration of different types of imDCs on day 6 by the Transwell migration assay in the presence of CCL19. Compared with the control group, the results showed that cotransfection of Ad-CCR7 and RelB-siRNA remarkably increased the migration of imDCs in the presence of CCL19 (Fig. 2A). DCs are an important type of APCs, which are essential for the initial reaction of naive T cells. To evaluate the biological modulatory capacity of RelB-siRNA on the lymphocyte proliferation response, the different types of imDCs were cocultured with T cells on day 7 to induce MLR. After 3 days, compared with the control group, cotransfection of Ad-CCR7 and RelB-siRNA significantly decreased the ability to stimulate T-cell proliferation after LPS treatment (Fig. 2B). These results indicated that CCR7 overexpression along with RelB KD accelerated the migration ability in imDCs and concurrently inhibited the proliferation response in $\mathrm{T}$ cells, thus inducing immune tolerance.

CCR7 overexpression and RelB KD in imDCs induced a shift from Th1 cells toward Th2 cells

Th1/Th2 cells are two subsets of T cells, which can restrict each other's function, and are important for the induction and maintenance of immune tolerance. It is widely accepted that the cytokines secreted by the $\mathrm{T}$ cells shape the outcome of the immune response [11]. We detected cytokines in the supernatant of the cocultured T cells of different types of imDCs. As shown in Fig. 3A, as compared with the control group, lower amounts of IFN- $\gamma$ or IL-2, but higher amounts of IL-4 or IL-10, were observed in imDCs after CCR7 overexpression and RelB KD. As expected, IL-2 or IFN- $\gamma$ was released by the Th1 cells, and IL-4 or IFN- $\gamma$ was released by the Th2 cells. At the same time, a lower proportion of CD4+ T cells produced IFN- $\gamma$, and an increased percentage synthesized Th2 cytokines in the T cells co-cultured with DCs previously transfected with siRNA RelB and CCR7 was observed in the flow cytometry (Fig.3C, Fig.3D ). T-bet and GATA-3 are specific transcription factors for Th1 and Th2 cells, respectively. To further support the observation, T-bet and GATA3 protein expressions were determined in the T cells cocultured with the different types of imDCs by immunoblot analysis. The results showed that $\mathrm{T}$ cells cocultured with imDCs transfected with Ad-CCR7 and RelB-siRNA showed a significant reduction in T-bet protein expression, whereas GATA3 protein expression was significantly upregulated (Fig. 3B), indicating that 
Fig. 3. CCR7 overexpression and RelB KD imDCs in imDCs induced a shift from Th1 toward Th2 cells (A) Supernatant was harvested after 96 h coculture with T cells. Cytokines in the supernatant were quantified by ELISA. Results showed that cotransfection of Ad-CCR7 and RelB-siRNA significantly decreased the amounts of IFN- $\gamma$ and IL- 2 while increasing the amounts of IL-4 and IL-10 in imDCs. ${ }^{* * *} \mathrm{P}<0.01$, compared with control group. (B) Western blot analysis of GATA-3 and T-bet expression in the $\mathrm{T}$ cells cocultured with different types of DCs. The expression of T-bet was significantly downregulated and GATA3 was upregulated in imDCs with CCR7 overexpression and RelB KD. ${ }^{* * *} \mathrm{P}<0.01$, compared with the control group. (C) FACS showed the frequency of INF-Y/ CD4+ T cells, which were depicted with characteristic dot plots. The statistical analyses showed that INF-Y/ CD4+ $\mathrm{T}$ was significantly elevated in that $\mathrm{T}$ cells cocultured with imDCs having CCR7 overexpression and RelB KD. ${ }^{* * *} \mathrm{P}<0.001$, as compared with the control group. (D) FACS showed the percent of IL-4/ CD4+ T cells, which were depicted with characteristic dot plots. The statistical analyses showed that Il-4/ CD4+ T was significantly elevated in that $\mathrm{T}$ cells cocultured with imDCs having CCR7 overexpression and RelB KD. $* * * \mathrm{P}<0.001$, as compared with the control group.

CCR7 overexpression along with RelB KD in imDCs was able to polarize a shift from Th1 toward Th2.

\section{CCR7 overexpression and RelB KD in imDCs induced Treg cells}

Several studies have shown that costimulatory signals are necessary for the generation of the CD4 + CD25+ Treg cells, which are considered important factors in the maintenance of transplantation tolerance [12]. To determine whether imDCs with CCR7 overexpression and RelB KD are capable of enhancing preservation of CD4 + CD25+ cells, we used flow cytometry to evaluate the amount of CD $4+$ CD25 + Treg cells in the T cells cocultured with different types of imDCs. As expected, compared with the other groups, an elevated number of CD4 + CD25 + T-cell fractions was observed in the T cells cocultured with imDCs having CCR7 overexpression and RelB KD (Fig. 4A). Moreover, FoxP3 is a crucial transcription factor

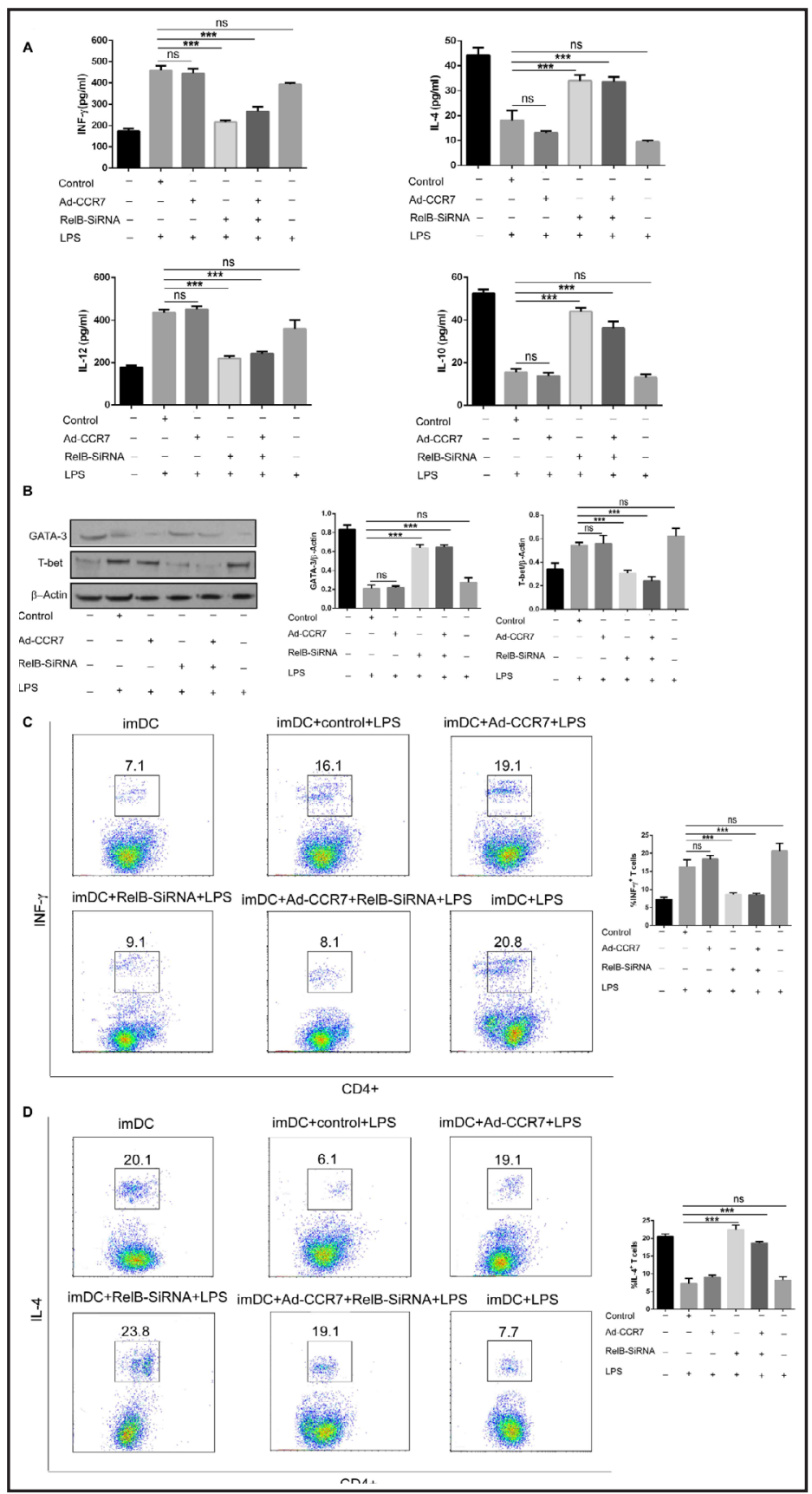




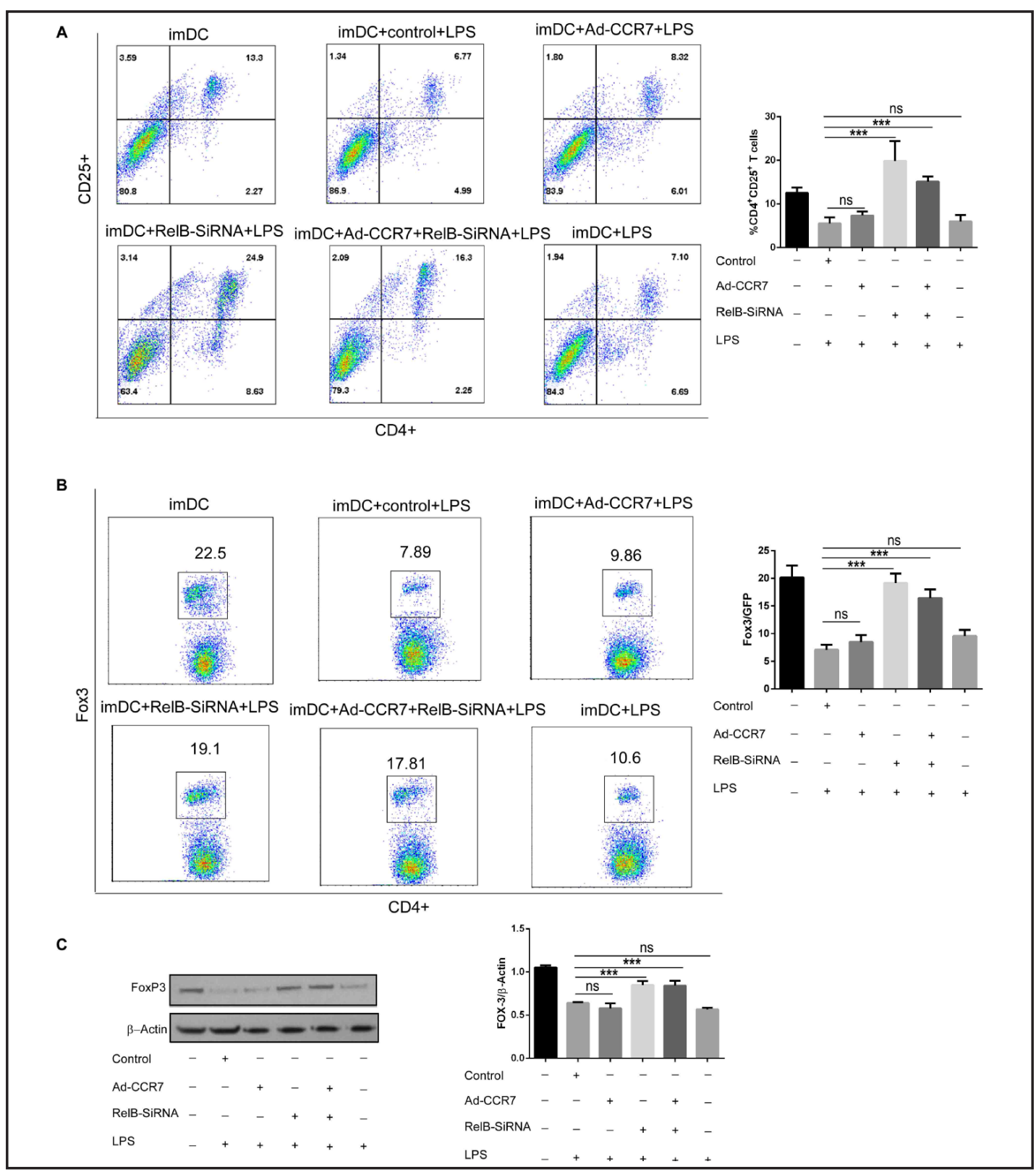

Fig. 4. CCR7 overexpression and RelB KD in imDCs induced more Treg cells for immune tolerance. (A) FACS analysis showed that cotransfection of Ad-CCR7 and RelB-siRNA significantly increased the number of CD4+CD25+T cells in comparison with the control group ( ${ }^{* * *} \mathrm{P}<0.01$ ). (B) FACS showed the frequency of Foxp3/ CD4+ T cells, which were depicted with characteristic dot plots. The statistical analyses showed that Foxp3 expression was significantly elevated in that T cells cocultured with imDCs having CCR7 overexpression and RelB KD. ${ }^{* * *} \mathrm{P}<0.001$, as compared with the control group. (C) Western blot analysis showed that Foxp3 expression was significantly elevated in that T cells cocultured with imDCs having CCR7 overexpression and RelB KD. ${ }^{* * *} \mathrm{P}<0.01$, compared with the control group.

for regulatory properties of T cells and is specifically expressed on the Treg cells. To further illuminate the mechanism of Treg cells, we also used Western blotting and flow cytometry to determine FoxP3 expression. As shown in Fig. 4B and 4C, FoxP3 protein levels were significantly elevated in T cells cocultured with imDCs having CCR7 overexpression and RelB $\mathrm{KD}$, supporting the assertion that this type of imDCs may facilitate immune tolerance by increasing the number of CD4+CD25+Foxp3+ Treg cells. 


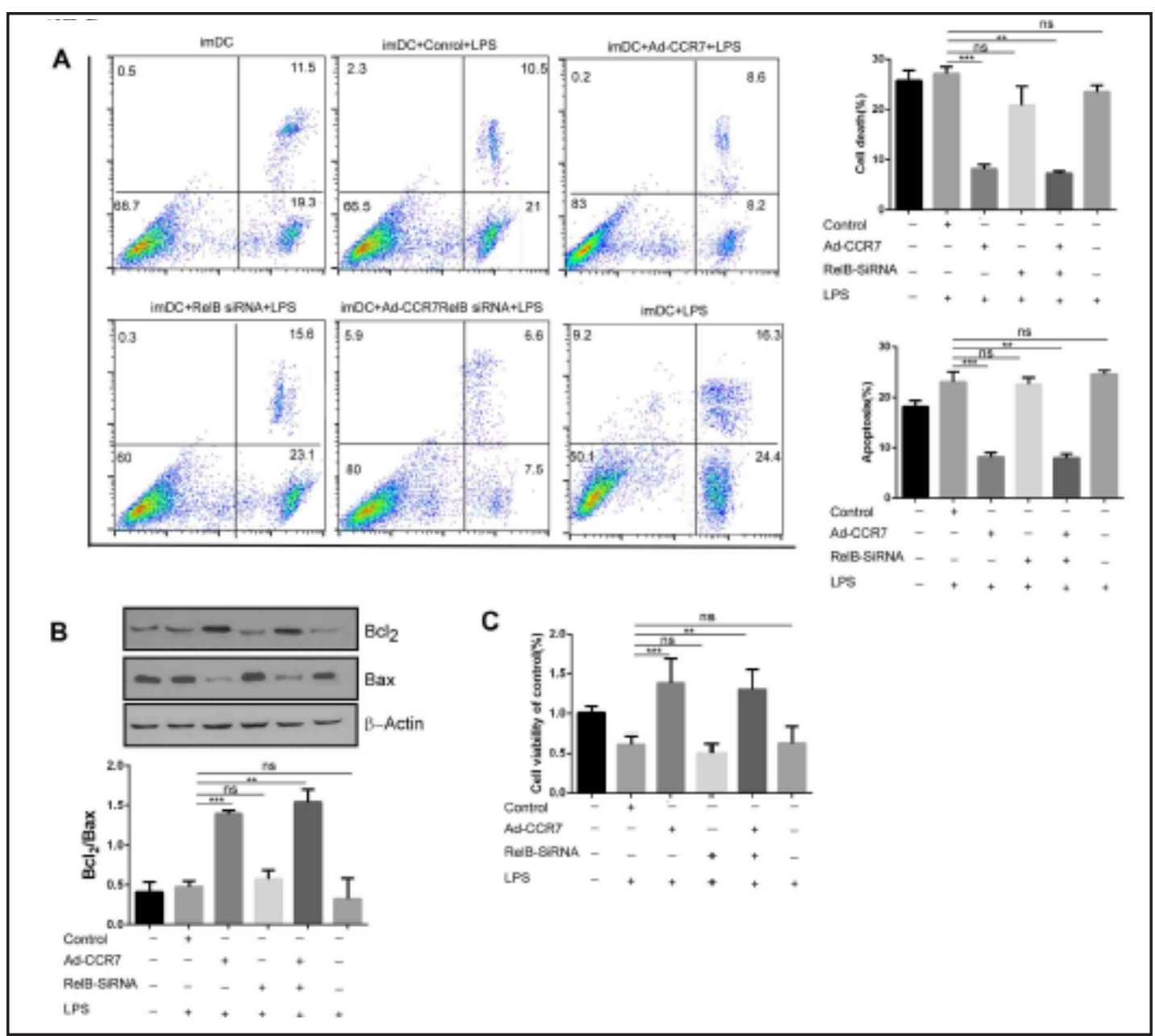

Fig. 5. Cotransfection of Ad-CCR7 and RelB-siRNA in imDCs possessed antiapoptotic ability and higher cell viability. (A) Apoptosis was depicted with scatter plots by Annexin V-FITC and PI staining. The results showed that cotransfection of Ad-CCR7 and RelB-siRNA significantly decreased the percentage of the apoptotic or dead imDCs compared with the control group ( ${ }^{* *} \mathrm{P}<0.01$, $\left.{ }^{* * *} \mathrm{P}<0.001\right)$. (B) Western blotting results showed the decreased ratio of $\mathrm{Bcl} 2$ and Bax in imDCs that were cotransfected with both Ad-CCR7 and RelB-siRNA. ${ }^{* * P}<0.01,{ }^{* * *} \mathrm{P}<0.001$, compared with the control group. (C) MTT assays showed that imDCs cotransfected with Ad-CCR7 and RelB-siRNA had higher cell viability. ${ }^{* *} \mathrm{P}<0.01$, compared with the control group.

imDCs with CCR7 overexpression and RelB KD possessed antiapoptosis and high cell migration viability.

CCR7 are not only potent chemokine receptors for DC cells, but have also been shown to inhibit apoptosis and promote the survival of DCs by certain signal pathways [13]. To determine whether imDCs with CCR7 overexpression and RelB KD have antiapoptosis for long-term survival, different types of imDCs were pretreated for $24 \mathrm{~h}$ in starvation media with CCL19 to induce cell apoptosis (see the Materials and Methods), following which we assessed the percentage of apoptotic or dead cells by flow cytometry. As shown in Fig. 5A, the percentage of the apoptosis or cell death in the control was greater than that in the group with imDCs having CCR7 overexpression and RelB KD. Furthermore, Western blotting showed that the apoptosis marker Bcl2 increased and Bax decreased in the cotransfected imDCs (Fig. 5B). These results indicated that CCR7 overexpression along with RelB KD protect the cells from apoptosis or death. After culturing for $24 \mathrm{~h}$ in starvation media, the MTT assay was used to inspect DC-cell viability. The cell viability of cotransfected imDCs was higher than that of the control (Fig. 5C). These results confirmed that imDCs with CCR7

\section{KARGER}




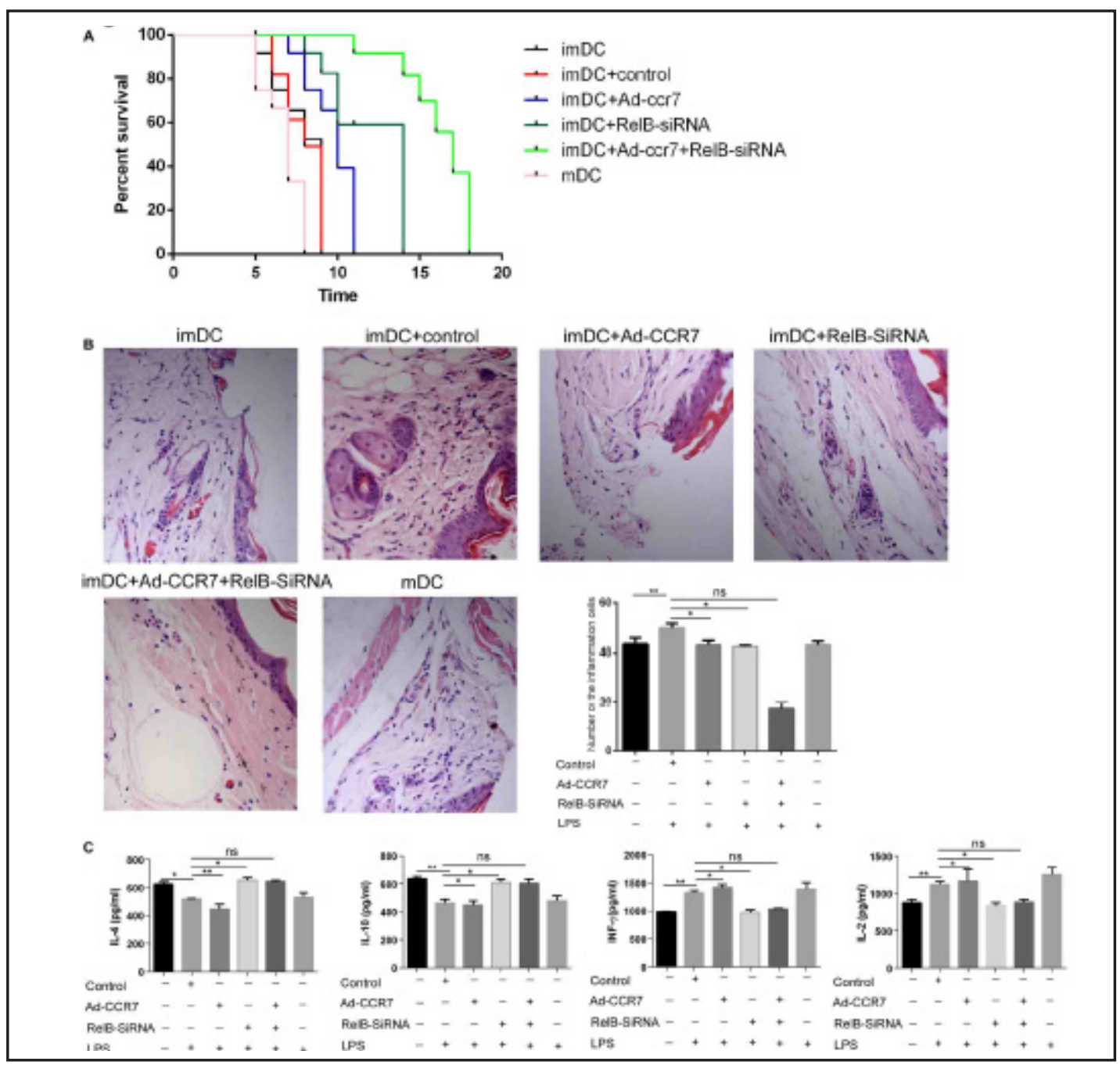

Fig. 6. Injection of CCR7 overexpression and RelB KD in imDCs prolonged skin allograft survival. A murine model of skin allograft transplantation was established using skin grafts derived from BALB/c donors, which were transplanted onto $\mathrm{C} 57 \mathrm{BL} / 6$ recipients. BALB/c recipient mice were treated with injections of $1 \times 10^{6}$ of different types of DCs. (A) Statistical analysis showed that the groups injected with the imDCs transfected with Ad-CCR7 and RelB-siRNA together, thereby significantly increased survival rate of the transplanted skin grafts, as compared with the control group. (B) Statistical analysis of the hematoxylin and eosin (H\&E) staining showed that the group injected with imDCs of cotransfection of Ad-CCR7 and RelB-siRNA had less substantial inflammatory-cell infiltration. ${ }^{*} \mathrm{P}<0.05$, ${ }^{* *} \mathrm{P}<0.01$, compared with the control group. (C) Serum levels of IL-2, IL-4, IL-10, and IFN- $\gamma$ from different mice were tested by Enzyme-linked immunosorbent assays (ELISA) on day 7 after skin transplantation. The levels of IFN- $\gamma$ and IL-2 were decreased in mice injected with imDCs having CCR7 overexpression and RelB KD, and in the meanwhile the level of IL-4 and IL-10 were elevated. ${ }^{*} \mathrm{P}<0.05,{ }^{* *} \mathrm{P}<0.01$, compared with the control group.

overexpression along with RelB KD have antiapoptotic ability and high cell viability, which resulted in longer survival of the imDCs, thus facilitating immune tolerance.

\section{CCR7 overexpression and RelB KD in imDCs prolonged skin allograft survival}

As CCR7 overexpression and RelB KD in imDCs were demonstrated to obtain the abilities of high migration, immune tolerance, and antiapoptosis in vitro, we further monitored their effect on skin allograft rejection in vivo. We established a murine model of skin allograft transplantation using skin grafts derived from BALB/c donors transplanted onto C57BL/6

\section{KARGER}




\section{Cellular Physiology Cell Physiol Biochem 2017;42:455-468 \\ \begin{tabular}{ll|l} 
DOI: 10.1159/000477593 & $\begin{array}{l}\text { O } 2017 \text { The Author(s). Published by S. Karger AG, Basel } \\
\text { www.karger.com/cpb }\end{array}$ \\
\cline { 2 - 4 } aublished online: May 31, 2017
\end{tabular}}

recipients, recording wound healing, skin color, and connectivity to evaluate the survival of transplanted skin. As shown in the Fig. 6A, either CCR7 overexpression or RelB KD alone in imDCs increased graft survival, but not as much as the concurrent CCR7 overexpression and RelB KD in imDCs, which indicates that concurrent CCR7 overexpression and RelB KD in imDCs remarkably prolongs the skin survival. To histologically investigate host immune reactions, an additional 30 mice were subjected to the same transplantation procedure to obtain skin tissue slides on day 10 after transplantation. Analysis revealed that full-thickness skin grafts from the control group were infiltrated by a mass of inflammatory cells, whereas the cotransfected imDC group showed less substantial inflammatory-cell infiltration (Fig. 6B). ELISA measurement showed that the cytokines IL-2 or IFN- $\gamma$ in the peripheral blood of the recipient mice injected with imDCs with CCR7 overexpression and RelB KD were lower than that from the mice injected with the control imDCs, whereas the secretion of cytokines IL-4 or IL-10 was higher (Fig. 6C). These results confirmed that injecting imDCs with CCR7 overexpression and RelB KD can prolong the skin allograft survival by decreasing the immune rejection, and may represent a promising method for potential therapeutic applications in skin transplantation.

\section{Discussion}

With regard to allogeneic skin transplantation, the lifespan of the transplanted graft skin may be short because of graft rejection. The rejection reaction is problematic for clinicians and causes constant difficulty during organ transplantation. Recent experimental evidence has demonstrated that gene-modified DCs have the ability to extend allograft survival beyond that which can be achieved through drug intervention or cytokine-induced Tol-DCs [14]. The induction of gene-modified Tol-DCs has been suggested as an alternative approach to alleviate immune rejection. In the present study, we transfected imDCs with AdCCR7 and RelB-siRNA together. As a result, we generated imDCs with CCR7 overexpression with a relatively high migration ability while maintaining a low expression of costimulatory molecules because of RelB KD, inhibiting T-cell responses and generating Treg cells, even with LPS stimulation. Furthermore, such imDCs possess antiapoptotic ability and demonstrated longer survival than other types of DCs. Finally, we also found that administration of such DCs significantly prevented allograft rejection and delayed skin allograft rejection in murine skin transplantation, suggesting the potential clinical use of gene-modified DC.

DC migration from injected sites to lymph nodes is an important requirement for effective immunization [15]. imDCs show low expression levels of costimulatory molecules, thereby laying the foundation for clinical application of imDCs in skin transplantation. However, the low CCR7 expression on DCs will affect the ability of the imDCs to induce immune tolerance [16]. In addition, it is difficult to control the environmental conditions and to guarantee that the administered DCs are in the immature stages when they reach the draining LNs. There is a great need to enhance the tolerogenic capacity of DCs and to eventually obtain DCs suitable for clinical applications. RelB plays a critical role in DC maturation and regulates the B7/ CD28 costimulatory signals, which play an important role in allograft rejection. To the best of our knowledge, the present study is the first study to demonstrate that the concurrent CCR7 overexpression and RelB KD induces tolerance in imDCs and prolong allograft survival in skin transplantation.

CCR7 is considered a key regulator of DC in trafficking from the periphery to the draining lymph node. An additional biological function of CCR7 is its involvement in the immune responses, which induces immune rejection [17]. Various recent reports have demonstrated that CCR7 facilitates DC maturation, thus enhancing T-cell priming to activate the T-cell response [18]. It was also reported CCR7 blockade led to improved graft survival in lowrisk corneal transplantation [10]. On the other hand, RelB is required for the steady-state accumulation of migratory DCs in draining LNs, and a deficiency of the alternative RelB/p52 signaling pathway was shown to decrease DC migration ability [19]. However, in the present 


\section{Cellular Physiology Cell Physiol Biochem 2017;42:455-468

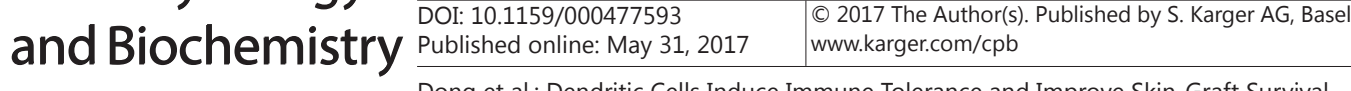

study, we found that even the imDCs with CCR7 overexpression maintain the immature status after LPS stimulation because of the low RelB expression. Meanwhile, the RelB-siRNA also cannot affect migration ability because of CCR7 overexpression, indicating that CCR7 and RelB cannot affect each other's function.

DC apoptosis was significantly associated with immune activity. The collective apoptosis and subsequent phagocytosis of the DC apoptotic bodies has been proposed as a possible mechanism for inducing immune response [20]. After reaching the lymph node, the short survival duration of imDCs does not permit these cells to perform their function of inducing immune tolerance. However, it has also been observed that exaggerated extension of DC lifespan leads to autoimmunity disease [21]. CCR7 promotes survival in mDCs by inhibiting apoptotic hallmarks of DCs, such as loss of mitochondrial membrane potential, membrane phosphatidylserine exposure, increased membrane blebs, and nuclear changes [22]. In the present study, we also found that concurrent CCR7 overexpression and RelB KD in imDCs obtained stronger antiapoptotic ability and expired significantly less than those of the other groups. However, whether such imDCs will remain alive for a longer duration in vivo requires further study.

CD4+CD25+Foxp3 Tregs, which constitute 5\%-10\% of peripheral CD4+ T cells, were considered to play a central role in inducing and maintaining tolerance by suppressing T-cell responses in several animal models and in a clinical trial of transplantation [23, 24]. The therapeutic potential of Tregs, termed cellular immunotherapy, has been shown to be effective for controlling immune responses in the graft-versus-host disease. Foxp3 appears to function as a master regulator in the development and function of regulatory $\mathrm{T}$ cells. In animal studies, administration of Foxp3-positive T cells has been shown to lead to marked reductions in the severity of immune rejection in some disease [25]. Consistent with previous studies, we observed that treatment with cells having CCR7 overexpression and RelB KD led to an increased number of CD $4+\mathrm{CD} 25+\mathrm{T}$ cells and increased foxp3 protein levels in the CD4+ T cells. This appeared to correlate with prolonged survival of skin allografts, suggesting that such imDCs possess a "tolerogenic" property, and would thus be useful in the treatment of immune rejection of the skin transplantation.

imDCs also induce suppressive elements through the secretion of several cytokines to induce immune tolerance. The cytokines promote changes to the DCs in phenotype and function, thereby influencing Th-cell differentiation [26, 27]. Th1/Th2 cells are two sets of $\mathrm{T}$ cells and restrict each other's function, which is critical for the balance between immune tolerance and rejection. In the immune system, the Th1/Th2-cell ratio is maintained in a dynamic balance, and it has been suggested that Th1 to Th2 switching may prevent the development of organ-specific autoimmune pathologies [28]. Consistent with previous reports, in the present study, the increased percentage of Th2, Treg and decreased percentage of Th1 among total T cells was achieved by co-culture with concurrent CCR7 overexpression and RelB KD imDCs. Furthermore, the upregulated Th2-related cytokines and downregulated Th1-related cytokines were also observed in vitro and in vivo, which may be one of the mechanisms by which concurrent CCR7 overexpression and RelB KD in imDCs induced immune tolerance.

More importantly, we showed that systemic administration of DCs with CCR7 overexpression and Rel KD can prolong skin allograft survival. However, the present study did not observe long-term survival of these skin grafts, which may have been associated with poor proliferative ability of imDCs, which cause them being unable to produce lasting effects. On the other hand, siRNA transfection is transient, lasting only for 1 week. In addition, immune reactions generated by injecting the imDCs intravenously affected the graft as well as the whole organism, causing serious side effects such as graft-versus-host-disease, infections (related to immunosuppression), and wound-healing difficulties. Furthermore, the mechanisms for regulating the immune reaction are complex, and it is possible that other proteins or pathways modify DC function. The approach of jointly inducing various mechanisms working together to achieve transplantation tolerance is an important direction 


\section{Cellular Physiology Cell Physiol Biochem 2017;42:455-468

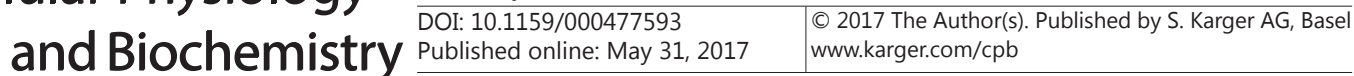 \\ Dong et al.: Dendritic Cells Induce Immune Tolerance and Improve Skin-Graft Survival}

for future research. Further research is required to identify a powerful and clinically practical tool for stable immune-tolerance induction, thereby enhancing the survival of skin.

In the present study, we generated concurrent CCR7 overexpression and RelB KD in DCs, which potentiated migration ability, maintained immune tolerance, and achieved antiapoptotic ability in vitro [29]. Importantly, concurrent CCR7 overexpression and RelB $\mathrm{KD}$ in imDCs leads to increased allograft survival, laying the foundation for experimental or clinical application of imDCs in skin allograft transplantation.

\section{Disclosure Statement}

The authors of this article declare no conflict of interests.

\section{References}

1 Ke T, Yang M, Mao D, Zhu M, Che Y, Kong D, Li C: Co-transplantation of skin-derived precursors and collagen sponge facilitates diabetic wound healing by promoting local vascular regeneration. Cell Physiol Biochem 2015;37:1725-1737.

-2 Penna G, Giarratana N, Amuchastegui S, Mariani R, Daniel KC, Adorini L: Manipulating dendritic cells to induce regulatory t cells. Microbes Infect 2005;7:1033-1039.

3 Xin HM, Peng YZ, Yuan ZQ Guo H: In vitro maturation and migration of immature dendritic cells after chemokine receptor 7 transfection. Can J Microbiol 2009;55:859-866.

4 Shi Q Yin Z, Liu P, Zhao B, Zhang Z, Mao S, Wei T, Rao M, Zhang L, Wang S: Cilostazol suppresses il-23 production in human dendritic cells via an ampk-dependent pathway. Cell Physiol Biochem 2016;40:499508.

-5 Guo M, Yan R, Wang C, Shi H, Sun M, Guo S, Xiao C: Ifn regulatory factor-1 modulates the function of dendritic cells in patients with acute coronary syndrome. Cell Physiol Biochem 2015;36:599-610.

-6 Thomas R: Relb and the aryl hydrocarbon receptor: Dendritic cell tolerance at the epithelial interface. Immunol Cell Biol 2013;91:543-544.

7 Luo L, Sun Z, Fang Q Huang S, Bai X, Luo G: Effects of tolerogenic dendritic cells generated by sirnamediated relb silencing on immune defense and surveillance functions of t cells. Cell Immunol 2013;282:28-37.

8 Martin E, O'Sullivan B, Low P, Thomas R: Antigen-specific suppression of a primed immune response by dendritic cells mediated by regulatory t cells secreting interleukin-10. Immunity 2003;18:155-167.

-9 Li M, Zhang X, Zheng X, Lian D, Zhang ZX, Ge W, Yang J, Vladau C, Suzuki M, Chen D, Zhong R, Garcia B, Jevnikar AM, Min WP: Immune modulation and tolerance induction by relb-silenced dendritic cells through rna interference. J Immunol 2007;178:5480-5487.

10 Hos D, Dorrie J, Schaft N, Bock F, Notara M, Kruse FE, Krautwald S, Cursiefen C, Bachmann BO: Blockade of ccr7 leads to decreased dendritic cell migration to draining lymph nodes and promotes graft survival in low-risk corneal transplantation. Exp Eye Res 2015;146:1-6.

11 Li JG, Du YM, Yan ZD, Yan J, Zhuansun YX, Chen R, Zhang W, Feng SL, Ran PX: Cd80 and cd86 knockdown in dendritic cells regulates th1/th2 cytokine production in asthmatic mice. Exp Ther Med 2016;11:878-884.

12 Lin J, Yang L, Silva HM, Trzeciak A, Choi Y, Schwab SR, Dustin ML, Lafaille JJ: Increased generation of foxp $3(+)$ regulatory $t$ cells by manipulating antigen presentation in the thymus. Nature Commun 2016;7:10562.

13 Escribano C, Delgado-Martin C, Rodriguez-Fernandez JL: Ccr7-dependent stimulation of survival in dendritic cells involves inhibition of gsk3beta. J Immunol 2009;183:6282-6295.

14 Tang J, Zhou X, Liu J, Meng Q, Han Y, Wang Z, Fan H, Liu Z: Il-25 promotes the function of cd4+cd25+t regulatory cells and prolongs skin-graft survival in murine models. Int Immunopharmacol 2015;28:931937.

15 Makdessi SA, Sweidan H, Schmid E, Weimar U, Gulbins E, Lang F: Quantitative determination of ceramide molecular species in dendritic cells. Cell Physiol Biochem 2016;39:1608-1617. 


\section{Cellular Physiology Cell Physiol Biochem 2017;42:455-468 \begin{tabular}{l|l} 
DOI: 10.1159/000477593 & Ond Biochemistry \\
Publisnec onnine:-1Vay 31, 2017 & $\begin{array}{l}\text { 2017 The Author(s). Published by S. Karger AG, Basel } \\
\text { www.karger.com/cpb }\end{array}$
\end{tabular} \\ Dong et al.: Dendritic Cells Induce Immune Tolerance and Improve Skin-Graft Survival}

16 Jin Y, Chauhan SK, Saban DR, Dana R: Role of ccr7 in facilitating direct allosensitization and regulatory t-cell function in high-risk corneal transplantation. Invest Ophthalmol Vis Sci 2010;51:816-821.

17 McNamee EN, Masterson JC, Veny M, Collins CB, Jedlicka P, Byrne FR, Ng GY, Rivera-Nieves J: Chemokine receptor ccr7 regulates the intestinal th1/th17/treg balance during crohn's-like murine ileitis. J Leukoc Biol 2015;97:1011-1022.

18 Ziegler E, Oberbarnscheidt M, Bulfone-Paus S, Forster R, Kunzendorf U, Krautwald S: Ccr7 signaling inhibits t cell proliferation. J Immunol 2007;179:6485-6493.

19 Baratin M, Foray C, Demaria O, Habbeddine M, Pollet E, Maurizio J, Verthuy C, Davanture S, Azukizawa H, Flores-Langarica A, Dalod M, Lawrence T: Homeostatic nf-kappab signaling in steady-state migratory dendritic cells regulates immune homeostasis and tolerance. Immunity 2015;42:627-639.

20 Yan J, Schmid E, Hosseinzadeh Z, Honisch S, Shumilina E, Fuchs J, Lang F: Impact of janus kinase 3 on cellular ca release, store operated $\mathrm{ca}(2+)$ entry and $\mathrm{na}(+) / \mathrm{ca}(2+)$ exchanger activity in dendritic cells. Cell Physiol Biochem 2015;36:2287-2298.

21 Chen M, Huang L, Wang J: Deficiency of bim in dendritic cells contributes to overactivation of lymphocytes and autoimmunity. Blood 2007;109:4360-4367.

-22 Kuwabara T, Tanaka Y, Ishikawa F, Kondo M, Sekiya H, Kakiuchi T: Ccr7 ligands up-regulate il-23 through pi3-kinase and nf-kappa b pathway in dendritic cells. J Leukoc Biol 2012;92:309-318.

23 Issa F, Hester J, Milward K, Wood KJ: Homing of regulatory t cells to human skin is important for the prevention of alloimmune-mediated pathology in an in vivo cellular therapy model. PloS one 2012;7:e53331.

24 Zhou Y, Salker MS, Walker B, Munzer P, Borst O, Gawaz M, Gulbins E, Singh Y, Lang F: Acid sphingomyelinase (asm) is a negative regulator of regulatory t cell (treg) development. Cell Physiol Biochem 2016;39:985995.

25 Zhang JG, Chen XJ, Liu T, Jiang SJ: Foxp3+ associated with the pro-inflammatory regulatory t and thelper 17 effector cells in asthma patients. Exp Ther Med 2016;12:2753-2758.

-26 Kim WS, Kim H, Kwon KW, Im SH, Lee BR, Ha SJ, Shin SJ: Cisplatin induces tolerogenic dendritic cells in response to tlr agonists via the abundant production of il-10, thereby promoting th2- and tr1-biased t-cell immunity. Oncotarget 2016;7:33765-33782.

27 Yang WY, Shao Y, Lopez-Pastrana J, Mai J, Wang H, Yang XF: Pathological conditions re-shape physiological tregs into pathological tregs. Burns \& trauma 2015;3

28 van Panhuys N, Klauschen F, Germain RN: T-cell-receptor-dependent signal intensity dominantly controls cd4(+) t cell polarization in vivo. Immunity 2014;41:63-74.

29 Zhou Y, Pasham V, Chatterjee S, Rotte A, Yang W, Bhandaru M, Singh Y, Lang F: Regulation of na(+)/h(+) exchanger in dendritic cells by akt1. Cell Physiol Biochem 2015;36:1237-1249. 